\title{
A Mathematical Approach on Representation of Competitions: Competition Cluster Hypergraphs
}

\author{
Sovan Samanta $\mathbb{D}^{1},{ }^{1}$ G. Muhiuddin $\mathbb{D}^{2},{ }^{2}$ Abdulaziz M. Alanazi, ${ }^{2}$ and Kousik Das $\mathbb{D}^{3}$ \\ ${ }^{1}$ Department of Mathematics, Tamralipta Mahavidyalaya, Tamluk, WB-721636, India \\ ${ }^{2}$ Department of Mathematics, University of Tabuk, Tabuk 71491, Saudi Arabia \\ ${ }^{3}$ Department of Mathematics, D. J. H. School, Dantan, WB-721451, India \\ Correspondence should be addressed to Sovan Samanta; ssamantavu@gmail.com
}

Received 27 March 2020; Revised 24 April 2020; Accepted 30 April 2020; Published 10 June 2020

Academic Editor: Jean Jacques Loiseau

Copyright (c) 2020 Sovan Samanta et al. This is an open access article distributed under the Creative Commons Attribution License, which permits unrestricted use, distribution, and reproduction in any medium, provided the original work is properly cited.

Social networks are represented using graph theory. In this case, individuals in a social network are assumed as nodes. Sometimes institutions or groups are also assumed as nodes. Institutions and such groups are assumed as cluster nodes that contain individuals or simple nodes. Hypergraphs have hyperedges that include more than one node. In this study, cluster hypergraphs are introduced to generalize the concept of hypergraphs, where cluster nodes are allowed. Sometimes competitions in the real world are done as groups. Cluster hypergraphs are used to represent such kinds of competitions. Competition cluster hypergraphs of semidirected graphs (a special type of mixed graphs called semidirected graphs, where the directed and undirected edges both are allowed) are introduced, and related properties are discussed. To define competition cluster hypergraphs, a few properties of semidirected graphs are established. Some associated terms on semidirected graphs are studied. At last, a numerical application is illustrated.

\section{Introduction}

Cohen introduced the concept of a competition graph [1] with application in an ecosystem which was related to the competition among species in a food web. If two species have at least one common prey, then there is a competition between them. Let $\vec{G}=(V, \vec{E})$ be a digraph, which corresponds to a food web. A vertex $x \in V$ represents a species in the food web, and an $\operatorname{arc}(\overrightarrow{x, s}) \in \vec{E}$ means $x$ preys on the species $s$. The competition graph $C(\vec{G})$ of a digraph $\vec{G}$ is an undirected graph $G=(V, E)$ which has the same vertex set and has an edge between two distinct vertices $x, y \in V$ if there exists a vertex $s \in V$ and $\operatorname{arcs}$ $(\overrightarrow{x, s}),(\overrightarrow{y, s}) \in \vec{E}$.

There are various research works on competition graphs. Roberts et al. studied that any graph with isolated vertices is the competition graph $[2,3]$, and the minimum number of such vertices is called the competition number. Opsut discussed the computation of competition numbers [4] of a graph. Kim et al. introduced the p-competition graph [5] and also the $p$ competition number [6]. Brigham et al. introduced the $\varnothing-$ tolerance graph as a generalization of $p$-competition [7]. Cho and Kim studied competition numbers [8] of a graph having one hole. Li and Chang proposed about a competition graph [9] with $h$ holes. Factor and Merz introduced the $(1,2)$-step competition graph [10] of a tournament and extended the $(1,2)$-step competition graph. But group competition along with the individual competition is not considered in these papers. This study developed the representations of an individual and group competition by cluster hypergraphs.

A hypergraph is a generalization of a graph in which any subset of a vertex set is an edge rather than two vertex sets. Especially, Berge [11, 12] introduced hypergraphs as a generalization of graph theory as follows.

Let $X=\left\{x_{1}, x_{2}, \ldots, x_{n}\right\}$ be a finite set and let $E=\left\{e_{1}, e_{2}, \ldots, e_{m}\right\}$ be a family of subsets of $X$ such that

$$
\begin{aligned}
e_{i} & \neq \phi, \quad(i=1,2, \ldots, m), \\
\bigcup_{\{i=1\}}^{m} e_{i} & =X .
\end{aligned}
$$

The pair $(X, E)$ is called a hypergraph with vertex set $X$ and hyperedge set $E$. The elements $\left\{x_{1}, x_{2}, \ldots, x_{n}\right\}$ of $X$ are 
vertices of hypergraph $H$, and the sets $\left\{e_{1}, e_{2}, \ldots, e_{m}\right\}$ are hyperedges of hypergraph $H$.

Burosch and Ceccherini [13] characterised cube hypergraphs, where each hyperedge contains three vertices. Bergen et al. [14] studied infection in hypergraphs. There are various research works on hypergraphs [15-29]. Sonntag and Teichert [30] defined hypertrees, and they extended the notion to competition hypergraphs [31] in another paper in 2004. But, group competition was ignored in the paper. Cluster hypergraphs have been introduced here to capture the notion of a cluster node.

Mixed graphs represent social networks accurately as there may be directed edges and undirected edges. Directed edges are the indication of influences, dominations, or followers while undirected edges are the indication of the opposite cases where individuals are connected but without following each other. The study of mixed graphs $[32,33]$ was started from 1970 where the edges are directed or undirected as follows: Adiga et al. studied the adjacency matrix [34] of mixed graphs. There are various research works related to matrices [35] and isomorphism [36] on mixed graphs. There are many applications of mixed graphs on social networks. For example, Facebook networks [37] allow mixed direction when if at least one friend follows another along with their friendship, then there are directed edges (for following) along with undirected edges (for friendship). In this sense, considering the mixed graph where the edges both directed and undirected are considered as semidirected graphs, we introduced the representation of competition of semidirected graphs as a special type of hypergraphs, called the competition cluster hypergraph where the nodes may be a cluster or a simple node. This study has the following contributions. In Section 2, semidirected graphs and their properties have been developed. In Section 3, cluster hypergraphs and related notions are introduced. The competition cluster hypergraphs and their classifications are analyzed in Section 4. At last, an application and conclusions are drawn in Section 5. Throughout this paper, mixed graphs and semidirected graphs are used synonymously.

\section{Semidirected Graph}

The definition of a semidirected graph is given as follows.

Definition 1 (see [32]). Let $V$ be a nonempty set of elements, called vertices or nodes. Also, let $E=E_{1} \cup \overrightarrow{E_{2}}$ where $E_{1} \subset V \times V$ is a set of unordered pairs of vertices, i.e., $E_{1}=$ $\{(u, v) \mid u, v \in V\}$, called a set of undirected edges, and $\overrightarrow{E_{2}} \subset V \times V$ is a set of ordered pair of vertices, $\overrightarrow{E_{2}}=\{\overrightarrow{(a, b)} \mid a, b \in V\}$, called a set of directed edges. Here, $G=\left(V, E_{1}, \overrightarrow{E_{2}}\right)$ is said to be a semidirected graph.

The preliminary terms are not defined in the literature. These terms are defined as follows. Incidence number which is related to the degree of a node is defined in the following.

Definition 2. The degree of a vertex $u$ is denoted as a triplet $D(u)=\left(d(u), d^{+}(u), d^{-}(u)\right)$ where $d(u)$ is the number of all incident edges of $u$ in $E_{1}, d^{+}(u)$ is the number of outdirected edges of $\overrightarrow{E_{2}}$ from the vertex $u$, and $d^{-}(u)$ is the number of in-directed edges of $\overrightarrow{E_{2}}$ towards the vertex $u$. Now, the incidence number of a vertex $u$ is denoted as in $(u)$ and defined in $(u)=d(u)+d^{+}(u)-d^{-}(u)$.

Example 1. The degree of a vertex $a$ of the graph, as shown in Figure 1, is given as $d(a)=(1,1,2)$. Now, the incidence number is in $(a)=1+1-2=0$.

A related property has been developed as follows.

Theorem 1. The sum of the incidence numbers in a semidirected graph is always even.

Proof. Let $G=\left(V, E_{1}, \overrightarrow{E_{2}}\right)$ be a semidirected graph with $n$ vertices. If in $(u), d(u), d^{+}(u)$, and $d^{-}(u)$ denote the incidence number, degree, in-degree, and out-degree of a vertex $u$, respectively, then $\operatorname{in}\left(v_{i}\right)=d\left(v_{i}\right)+d^{+}\left(v_{i}\right)-d^{-}\left(v_{i}\right)$ and hence

$$
\sum_{i=1}^{n} \operatorname{in}\left(v_{i}\right)=\sum_{i=1}^{n} d\left(v_{i}\right)+\sum_{i=1}^{n} d^{+}\left(v_{i}\right)-\sum_{i=1}^{n} d^{-}\left(v_{i}\right) .
$$

Since one undirected edge contributes two degrees, then $\sum_{i=1}^{n} d\left(v_{i}\right)=$ even number. Also, if there is a directed edge in the graph, then it is a source of one vertex and also a sink of another vertex, thus $\sum_{i=1}^{n} d^{+}\left(v_{i}\right)=\sum_{i=1}^{n} d^{-}\left(v_{i}\right)$.

Hence, $\sum_{i=1}^{n}$ in $\left(v_{i}\right)=$ even number is true.

The definitions of complete semidirected graphs are introduced in the following.

Definition 3. If there exist all three types of connections, i.e., out-directed edges, in-directed edges, and undirected edges, between every pair of vertices, then the graph is called a complete semidirected graph.

Without having all three types of edges, sometimes graphs may be completely depending on the incidence number and connections between every pair of vertices.

Definition 4. A semidirected graph is said to be a completeincidence semidirected graph if every pair of vertices is connected by at least one edge (undirected or directed), and the incidence number of all vertices is equal.

Example 2. Since, as shown in Figure 2, there are connections (undirected or directed) between every pair of vertices and the incidence number of each of the vertices is 3 , it is a complete-incidence semidirected graph.

Definition 5. Neighbourhood, out-neighbourhood, and inneighbourhood of a vertex $u$ in a semidirected graph $G=$ $\left(V, E_{1}, \overrightarrow{E_{2}}\right)$ are denoted as $N(u), N^{+}(u)$, and $N^{-}(u)$ and defined as follows:

$$
\begin{aligned}
N(u) & =\left\{v \in V \mid(u, v) \in E_{1}\right\}, \\
N^{+}(u) & =\left\{v \in V \mid \overrightarrow{(u, v)} \in \overrightarrow{E_{2}}\right\}, \\
N^{-}(u) & =\left\{v \in V \mid \overrightarrow{(v, u)} \in \overrightarrow{E_{2}}\right\} .
\end{aligned}
$$




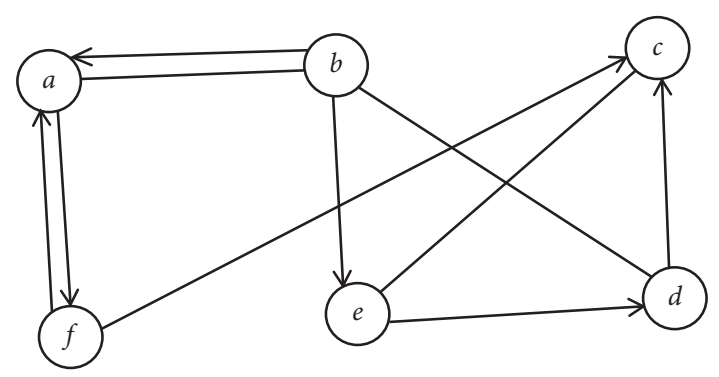

FIGURE 1: A semidirected graph.

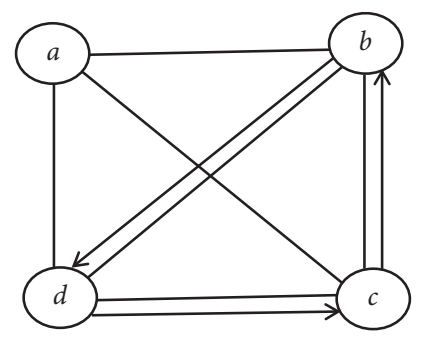

Figure 2: An example of a complete-incidence semidirected graph.

The maximal out-neighbourhood vertex set is an outneighbourhood of a vertex which is not contained in other out-neighbourhood of any vertices.

The maximal in-neighbourhood vertex set is an inneighbourhood of a vertex which is not contained in other in-neighbourhood of any vertices.

Example 3. In Figure 3,

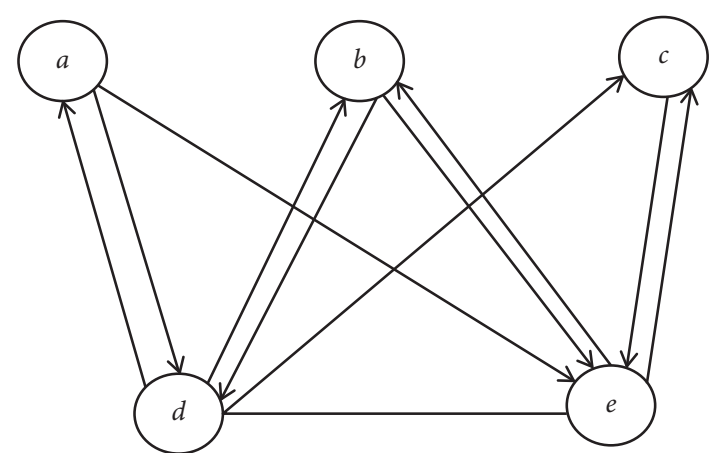

FIGURE 3: A semidirected graph.

$$
\begin{aligned}
N(d) & =\{e\}, \\
N^{+}(d) & =\{a, b, c\}, \\
N^{+}(e) & =\{b, c\}, \\
N^{-}(d) & =\{a, b\}, \\
N^{-}(e) & =\{a, b, c\} .
\end{aligned}
$$

Thus, $N^{+}(d)$ is a maximal out-neighbourhood set and $N^{-}(e)$ is a maximal in-neighbourhood set of the semidirected graph assumed in Figure 3.

Definition 6. The $m$-stepneighbourhood, $m$-step outneighbourhood, and $m$-step in-neighbourhood of a vertex $u$ in a semidirected graph $G=\left(V, E_{1}, \overrightarrow{E_{2}}\right)$ are denoted as $N_{m}(u), N_{m}^{+}(u)$, and $N_{m}^{-}(u)$ and defined as follows:

$$
\begin{aligned}
& N_{m}(u)=\left\{v_{m} \in V: \text { for all paths such that } u-v_{1}-v_{2}-\cdots-v_{m}\right\}, \\
& N_{m}^{+}(u)=\left\{v_{m} \in V: \text { for all paths such that } u \longrightarrow v_{1} \longrightarrow v_{2} \longrightarrow \cdots \longrightarrow v_{m}\right\}, \\
& N_{m}^{-}(u)=\left\{v_{m} \in V: \text { for all paths such that } u \longleftarrow v_{1} \longleftarrow v_{2} \longleftarrow \cdots \longleftarrow v_{m}\right\} .
\end{aligned}
$$

Example 4. In Figure 3, $N_{2}(d)=\{\varnothing\}, N_{2}^{+}(d)=\{e\}$, and $N_{2}^{-}(d)=\{e\}$.

Definition 7. The $m$-step semidirected graph $G_{m}=\left(V^{m}\right.$, $\left.E_{1}^{m}, \overrightarrow{E_{2}^{m}}\right)$ of a semidirected graph $G=\left(V, E_{1}, \overrightarrow{E_{2}}\right)$ is defined as follows:

(i) Vertex set of $G_{m}$ is $V^{m}=V$

(ii) Edge set of $G_{m}$ is $E_{1}^{m}=\left\{\left(u, v_{m}\right): v_{m} \in N_{m}(u)\right\}, \vec{E}_{2}^{m}=$ $\left\{\overrightarrow{\left(u, v_{m}\right)}: v_{m} \in N_{m}^{+}(u)\right.$ or $\left.N_{m}^{-}(u)\right\}$

Example 5. In Figure 4, the corresponding 2-step semidirected graph of Figure 3 is shown.

\section{Cluster Hypergraphs}

Definition 8 . Let $X$ be a nonempty set and $V_{X}$ be a subset of $P(X)$ such that $\phi \notin V_{X}$ and $X \subset V_{X}$. Now, $E$ be a multiset whose elements belong to $P(P(X))$ such that (i) $\phi \notin E$

(ii) For each element $e \in E$, there exists at least one element $v \in V_{X}$ such that $v \in e$

Then, $G=\left(V_{X}, E\right)$ is said to be cluster hypergraph where $V_{X}$ is said to be the vertex set and $E$ is said to be the multihyperedge set.

The $k$-cluster hypergraph is defined as follows.

Definition 9. Let $X$ be a nonempty set and $V_{X}$ be a subset of $P^{k}(X), k=1,2,3, \ldots$ such that $\phi \notin V_{X}$ and $X \subset V_{X}$. Now, $E$ be a multiset whose elements belong to $P\left(V_{X}\right)$ such that

(i) $\phi \notin E$

(ii) For each element $e \in E$, there exists at least one element $v \in V_{X}$ such that $v \in e$

Then, $G=\left(V_{X}, E\right)$ is said to be k-cluster hypergraph where $V_{X}$ is said to be the vertex set and $E$ is said to be the 


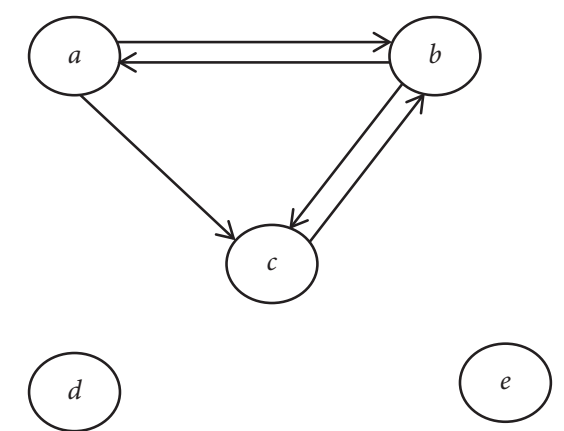

FIGURE 4: 2-step semidirected graph of Figure 3.

multi-hyperedge set. Generally, for $k=1,1$-cluster hypergraphs are assumed as cluster hypergraphs.

Example 6. In Figure 5, $X=\{A, B, C, E, F, G\}, V_{X}=\{\{A\}$, $\{B\},\{C\}, D=\{B, C\},\{E\},\{F\},\{G\}, H=\{F, G\}\}$ and $E=$ $\{\{\{A\},\{B\}\},\{\{A\},\{E\}\},\{\{B, C\},\{B\}\},\{\{B, C\},\{C\}\},\{\{F\},\{G\}\}$, $\{\{F, G\},\{F\}\},\{\{F, G\},\{G\}\},\{\{F, G\},\{B, C\}\}\}$. It can be easily verified that for each element $e$ in $E$, there exists an element $v \in V_{X}$ such that $v \in e$. For example, for $\{\{F, G\},\{F\}\}$ in $E$, there exists an element

$$
\{F, G\} \in V_{X}, \quad \text { such that }\{F, G\} \in\{\{F, G\},\{F\}\} .
$$

Remark 1.

(i) The vertex set of a cluster hypergraph may contain a group of people/individuals in a network as a node (cluster node) while all the people in the network are assumed as simple nodes. This concept is helpful to assume any organisation or group as nodes in any network. Also, it is assumed that each node inside a cluster node is automatically connected to the cluster node, but these inside nodes may not be connected to each other.

(ii) The definition of a cluster hypergraph includes the concept of the multi-hyperedge set. Thus, the repetition of elements in $E$ is allowed.

(iii) In a virtual representation (Figure 6) of any cluster hypergraph, the cluster nodes are assumed as separate nodes, and the connections to the inside nodes are shown in the representation.

3.1. Types of Cluster Hypergraphs. Depending on the cluster node sizes and their edges, cluster hypergraphs are classified into different categories. To classify, maximal nodes are to be defined. The maximal nodes are those nodes which are not contained in any other cluster nodes. The elements of $X$ are termed as simple nodes. A simple node may be termed as a maximal node if it does not belong to any other nodes. For example, in Figure 7 , the node $f$ is a simple as well as a maximal node, but the node $\{\{a, b\}, c, d\}$ is a maximal node.

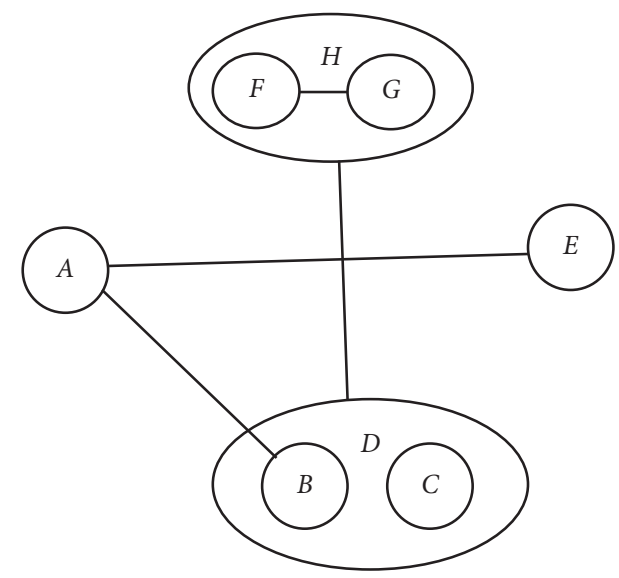

Figure 5: A cluster hypergraph.

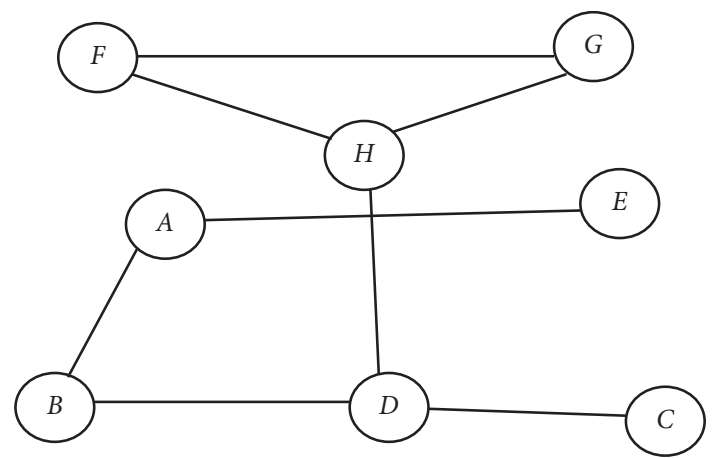

Figure 6: Virtual representation of Figure 5.

3.1.1. Uniform Cluster Hypergraphs. A cluster hypergraph is said to be a $(m, n)$-uniform cluster hypergraph if every edge of the hypergraph contains exactly $m$ nodes and each maximal node contains $n$ simple nodes. In Figure 8, a $(2,3)$-uniform cluster hypergraph is shown.

3.1.2. Cluster Connected Cluster Hypergraphs (CCCH). A cluster hypergraph is said to be cluster connected cluster hypergraphs $(\mathrm{CCCH})$ if there are edges only connecting the maximal cluster nodes. In that case, maximal cluster nodes are automatically connected to its internal nodes. In Figure 7, a $\mathrm{CCCH}$ is shown.

3.1.3. Completeness Property of Cluster Hypergraphs. Let $X$ be a nonempty set containing $n$ elements. A cluster hypergraph $G=(V, E)$ on $X$ contains maximum $|P(X)-\phi|$ number of vertices, i.e., $|V|=2^{n}-1$, and for the complete cluster hypergraph, the number of edges is $\mid P(P(X)-\phi)$ $-\phi \mid=2^{2^{n}-1}-1$. The completeness properties of different types of cluster graphs are discussed as follows.

(1) Complete CCCH. A complete $\mathrm{CCCH}$ is a cluster hypergraph where any two maximal nodes are connected by an edge. An example of a complete $\mathrm{CCCH}$ is shown in Figure 9. 

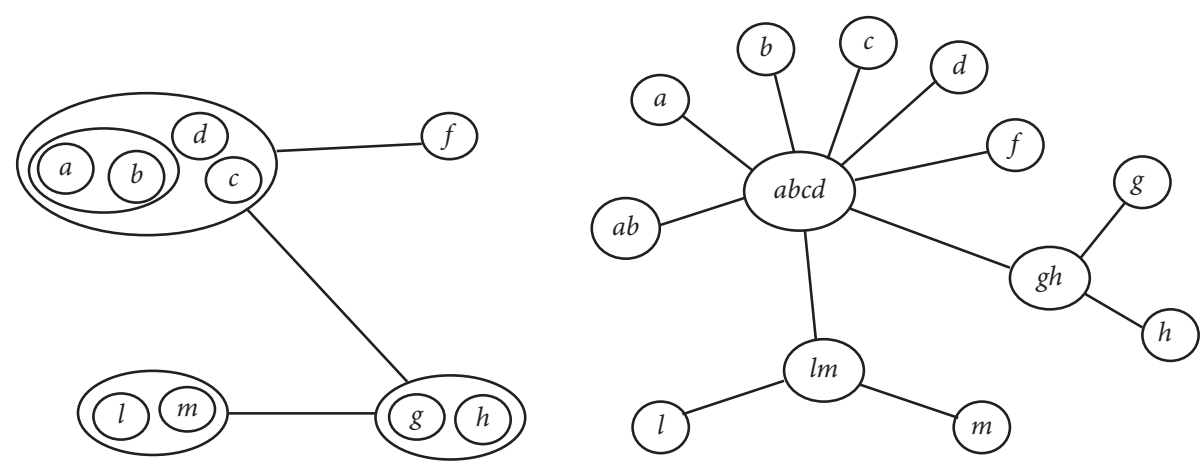

FIGURE 7: Virtual representations of CCCH (2-cluster hypergraphs have been assumed here).
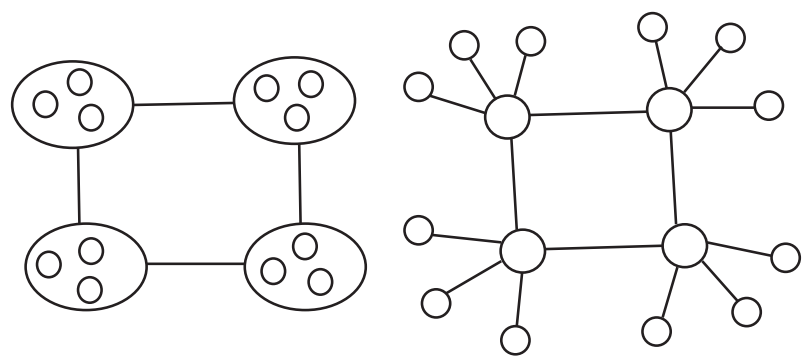

FIGURE 8: A (2,3)-uniform cluster hypergraph and its virtual representation.
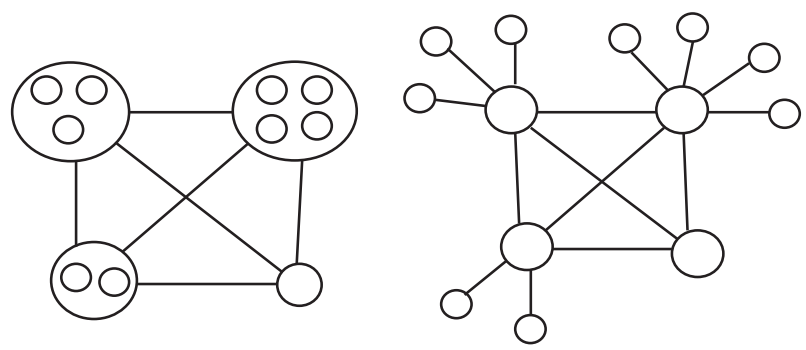

FIgURE 9: A complete CCCH and its virtual representation.

(2) Complete Uniform Cluster Hypergraph. A complete uniform cluster hypergraph is a $(m, n)$-uniform cluster hypergraph where an edge connects any two maximal nodes and an edge connects any two simple nodes within cluster nodes. An example of a complete (2,3)-uniform cluster hypergraph is shown in Figure 10.

Theorem 2. A complete $(m, n)$-uniform cluster hypergraph having $x$ cluster nodes contains $x \times{ }^{n} C_{m}+{ }^{x} C_{m}$ edges.

Proof. Let us consider a complete $(m, n)$-uniform cluster hypergraph having $x$ cluster nodes. The hypergraph has $x$ cluster nodes containing $n$ nodes per cluster. Thus, the total number of simple nodes is $x \times n$. Also, the graph is complete. Therefore, each edge contains exactly $m$ nodes. Hence, the total number of edges per cluster is ${ }^{n} C_{m}$. Also, the total number of edges among $x$ clusters is ${ }^{x} C_{m}$. Thus, the number of edges in the complete $(m, n)$-uniform cluster hypergraph having $x$ cluster nodes is $x \times{ }^{n} C_{m}+{ }^{x} C_{m}$.

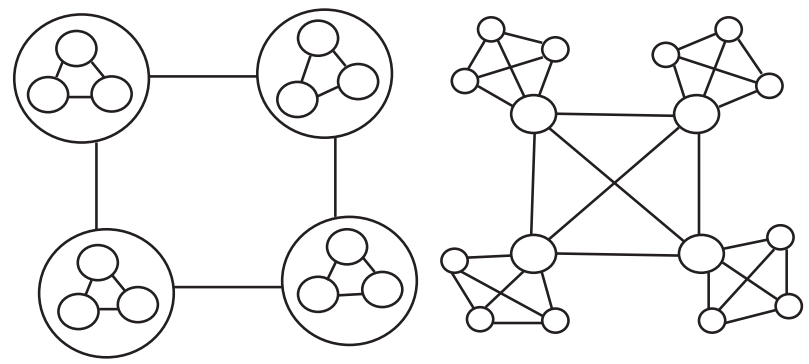

FIgURE 10: A complete uniform $\mathrm{CCCH}$ and its virtual representation.

Note. A $(m, n)$-uniform complete $\mathrm{CCCH}$ having $x$ cluster nodes contains ${ }^{x} C_{m}$ and $x \times n$ simple nodes.

\section{Competition Cluster Hypergraphs}

Competition cluster hypergraphs of semidirected graphs are defined where adjacent vertices by undirected edges form a cluster. If these adjacent vertices have common out-directed neighbours, then these vertices are also adjacent in competition cluster hypergraphs. The formal definition is given as follows.

Definition 10. Let $G=\left(X, E_{1}, \overrightarrow{E_{2}}\right)$ be a semidirected graph where $X$ is a nonempty vertex set, $E_{1}$ is the set of undirected edges, and $\overrightarrow{E_{2}}$ is the set of directed edges. Now, the competition cluster hypergraph of $G$ is denoted as $C(G)=\left(V_{X}, E\right)$ where $V_{X} \subset P(X)$ is the vertex set of $C(G)$ such that $X \subset V_{X}, \quad\left\{x_{i}, x_{j}\right\} \in V_{X}$ if $\left(x_{i}, x_{j}\right) \in E_{1}$ and $\left\{x_{1}, x_{2}, \ldots, x_{m}\right\} \in V_{X}$ if $\left\{x_{1}, x_{2}, \ldots, x_{m}\right\}$ forms a maximal clique in $G$ and $E$ which is the hyperedge set if there exists an edge containing vertices $x_{1}, x_{2}, \ldots, x_{m}$ if $N^{+}\left(x_{1}\right) \cap N^{+}\left(x_{2}\right) \cap \cdots \cap N^{+}\left(x_{m}\right) \neq \phi$ and $m=2, \ldots,|X|$.

Example 7. Let us consider a semidirected graph, as shown in Figure 11(a). The corresponding competition cluster hypergraph is shown in Figure 11(b). In the semidirected graph, $X=\{a, b, c, d, e, f\}$, and also $\{e, f, c\}$ forms a clique. So, the vertex set of the corresponding competition hypergraph is $V_{X}=X \cup\{e, f, c\}$. Now, $N^{+}(a) \cap N^{+}(b)=$ $\{a\}$ and $N^{+}(a) \cap N^{+}(b) \cap N^{+}(c)=\{d\}$, Thus, the edge set of $C(G)$ is $E=\{a b, a b c\}$. 


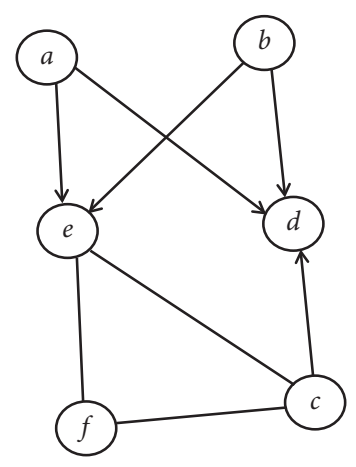

(a)

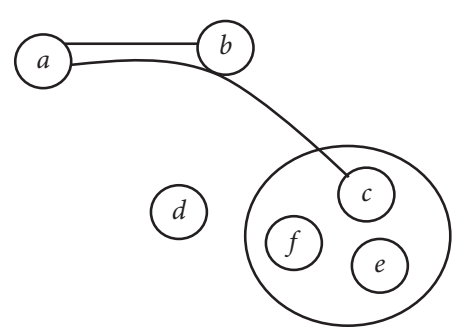

(b)

Figure 11: A competition cluster hypergraph. (a) A semidirected graph G. (b) Competition cluster hypergraphs of G.

Proposition 1. Let $G=\left(X, E_{1}, \vec{E}_{2}\right)$ be a semidirected graph and the corresponding competition cluster hypergraph of $G$ be $C(G)=\left(V_{X}, E\right)$. The number of edges in $C(G)$ is equal to the number of a maximal in-degree set of vertices in $G$ with cardinality greater than one.

Proof. Let $G=\left(X, E_{1}, \vec{E}_{2}\right)$ be a semidirected graph where $X$ is a nonempty vertex set and the corresponding competition cluster hypergraph of $G$ be $C(G)=\left(V_{X}, E\right)$. In competition cluster hypergraphs, an edge exists between two vertices $x$ and $y$ if they have a common vertex (or vertices). Along with this, if it is found that the third vertex, say $z$, has the same common vertex, then that edge will contain all the three vertices $x, y$, and $z$ and so on. Thus, the number of edges in $C(G)$ is equal to the number of a maximal in-degree set of vertices in $G$ with cardinality greater than one.

Remark 2. Let $G=\left(X, E_{1}, \vec{E}_{2}\right)$ be a semidirected graph and the corresponding competition cluster hypergraph of $G$ be $C(G)=\left(V_{X}, E\right)$. The number of cluster nodes in $C(G)$ is equal to the number of cliques by undirected edges in $G$.

Theorem 3. Let $G=\left(X, E_{1}, \vec{E}_{2}\right)$ be a semidirected graph and the corresponding competition cluster hypergraph of $G$ be $C(G)=\left(V_{X}, E\right)$. The number of maximal nodes in $C(G)$ is equal to a number of nodes which are not adjacent to other vertices by undirected edges in $G+$ (the number of undirected edges which are not part of any cliques in $G)+($ number of maximal cliques in $G$ ).

Proof. Let $G=\left(X, E_{1}, \vec{E}_{2}\right)$ be a semidirected graph where $X$ is a nonempty vertex set and the corresponding competition cluster hypergraph of $G$ be $C(G)=\left(V_{X}, E\right)$.

Case 1. $\left(E_{1}=\phi\right)$ : if the graph $G$ has no undirected edges, then every node in $C(G)$ is a simple node. Hence, the statement is obvious.

Case 2. $\left(E_{1} \neq \phi\right)$ : in this case, $G$ contains undirected edges. Those edges may construct maximal cliques or simple undirected edges. One maximal clique in $G$ will correspond to one cluster node in $C(G)$. The undirected edges which are not in any cluster also correspond to cluster nodes containing two simple nodes. Hence, the number of maximal nodes in $C(G)$ is equal to the number of nodes which are not adjacent to other vertices by undirected edges in $G+$ (the number of undirected edges which are not part of any cliques in $G)+($ number of maximal cliques in $G)$.

Corollary 1. Let $G=\left(X, E_{1}, \overrightarrow{E_{2}}\right)$ be a semidirected graph and the corresponding competition cluster hypergraph of $G$ be $C(G)=\left(V_{X}, E\right)$. All cliques in $G$ correspond to maximal nodes if the cliques are disjoint completely, i.e., no vertex and edges are common.

Definition 11 (double competition cluster hypergraphs). Let $G=\left(X, E_{1}, \overrightarrow{E_{2}}\right)$ be a semidirected graph where $X$ is a nonempty vertex set, $E_{1}$ is the set of undirected edges, and $\overrightarrow{E_{2}}$ is the set of directed edges. Now, the competition cluster hypergraph of $G$ is denoted as $C(G)=\left(V_{X}, E\right)$ where $V_{X} \subset P(X)$ is the vertex set of $C(G)$ such that $X \subset V_{X}$, $\left\{x_{i}, x_{j}\right\} \in V_{X}$ if $\left(x_{i}, x_{j}\right) \in E_{1}$ and $\left\{x_{1}, x_{2}, \ldots, x_{m}\right\} \in V_{X}$ if $\left\{x_{1}, x_{2}, \ldots, x_{m}\right\}$ forms a maximal clique in $G$ and $E$ which is the edge set if there exists an edge containing vertices $x_{1}, x_{2}, \ldots, x_{m}$ if $N^{+}\left(x_{1}\right) \cap N^{+}\left(x_{2}\right) \cap \cdots \cap N^{+}\left(x_{m}\right) \neq \phi$ and $m=2, \ldots,|X|$.

Example 8. A semidirected graph is shown in Figure 12(a). The corresponding double competition cluster hypergraph is shown in Figure 12(b).

Definition 12. ( Let $G=\left(X, E_{1}, \overrightarrow{E_{2}}\right)$ be a semidirected graph where $X$ is a nonempty vertex set, $E_{1}$ is the set of undirected edges, and $\overrightarrow{E_{2}}$ is the set of directed edges. Now, the $m$-step competition cluster hypergraph of $G$ is denoted as $C_{m}(G)=\left(V_{X}, E\right)$ where $V_{X} \subset P(X)$ is the vertex set of $C_{m}(G)$ such that $X \subset V_{X}, \quad\left\{x_{i}, x_{j}\right\} \in V_{X}$ if $\left(x_{i}, x_{j}\right) \in E_{1}$ and $\left\{x_{1}, x_{2}\right.$, $\left.\ldots, x_{n}\right\} \in V_{X}$ if $\left\{x_{1}, x_{2}, \ldots, x_{n}\right\}$ forms a maximal clique in $G$ and $E$ which is the hyperedge set if there exists an edge containing vertices $x_{1}, x_{2}, \ldots, x_{n}$ if $N_{m}^{+}\left(x_{1}\right) \cap$ $N_{m}^{+}\left(x_{2}\right) \cap \cdots \cap N_{m}^{+}\left(x_{n}\right) \neq \phi$ and $n=2, \ldots,|X|$.

Example 9. Let us consider a semidirected graph, as shown in Figure 3. The corresponding 2-step competition cluster 


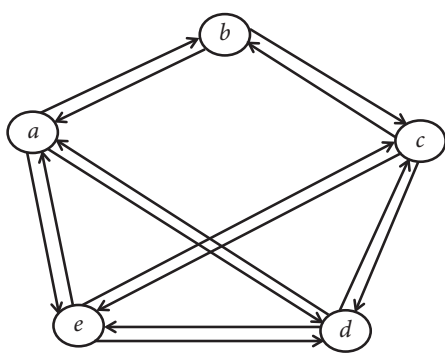

(a)

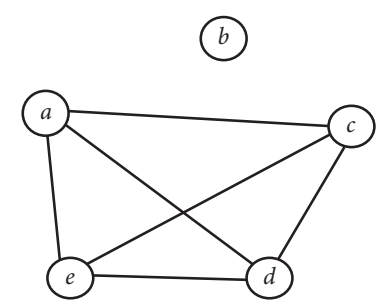

(b)

FIGURE 12: Double competition cluster hypergraphs. (a) A semidirected graph. (b) Double competition cluster hypergraph.

hypergraph $C_{m}(G)$ is shown in Figure 13. In the semidirected graph, $X=\{a, b, c, d, e\}$, and also $(d, e) \in E_{1}$. So, the vertex set of the corresponding 2-step competition hypergraph is $V_{X}=X \cup\{d, e\}$. Now, $N_{2}^{+}(a) \cap N_{2}^{+}(c)=\{b\}$. Thus, the edge set of $C_{m}(G)$ is $E=\{a c\}$.

Definition 13. A node in a cluster hypergraph is called an isolated node if there exist no edge to the node from the nodes of the graph. There are two types of isolated nodes.

(i) Isolated Maximal Node. A maximal node (simple or cluster) is called a maximal isolated node if it has no incident edges, i.e., it is isolated from all other maximal nodes of the cluster hypergraph. Consider a cluster hypergraph as shown in Figure 14. We observe that $\{k\}, D=\{\{g\},\{h\}\}$ are isolated maximal nodes.

(ii) Isolated Node in a Cluster. A simple node may be isolated within a cluster node of a cluster hypergraph. Consider a cluster hypergraph as shown in Figure 15 . We observe that only node $\{c\}$ is an isolated node in the cluster $F=\{\{c\},\{b\}\}$ of the cluster hypergraph.

Definition 14. Consider a cluster hypergraph $G$. Then, the competition number $k$ of $G$ is the minimum number of $k$ maximal isolated nodes with $G$ which forms the competition graph of a semidirected graph.

Example 10. Consider a cluster hypergraph (Figure 16) $G=$ $\left(V_{X}, E\right)$ where $V_{X}=\{\{a\},\{b\},\{a, b\},\{c\}\}$ and $E=\{\{\{c\},\{b\}\}\}$. Then, the corresponding semidirected graph (Figure 17) is drawn. There is no isolated node. Hence, the competition number of $G$ is 0 .

Algorithm 1. The steps to find the competition number of a cluster hypergraph are given as follows:

Step 1. Consider a cluster hypergraph $G$

Step 2. Draw the required directed or undirected edges to fit a corresponding semidirected graph $G^{\prime}$ from $G$

Step 3. The extra nodes if needed to get $C\left(G^{\prime}\right)=G$ may be taken as isolated nodes

Step 4. The minimum number of maximal isolated nodes will be the competition number of $G$

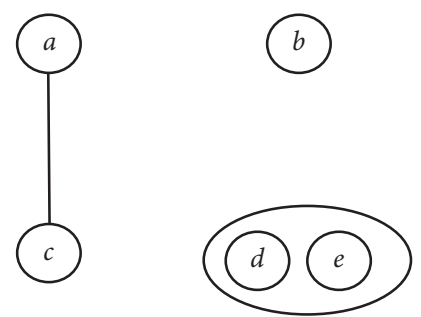

Figure 13: 2-step competition cluster hypergraph.

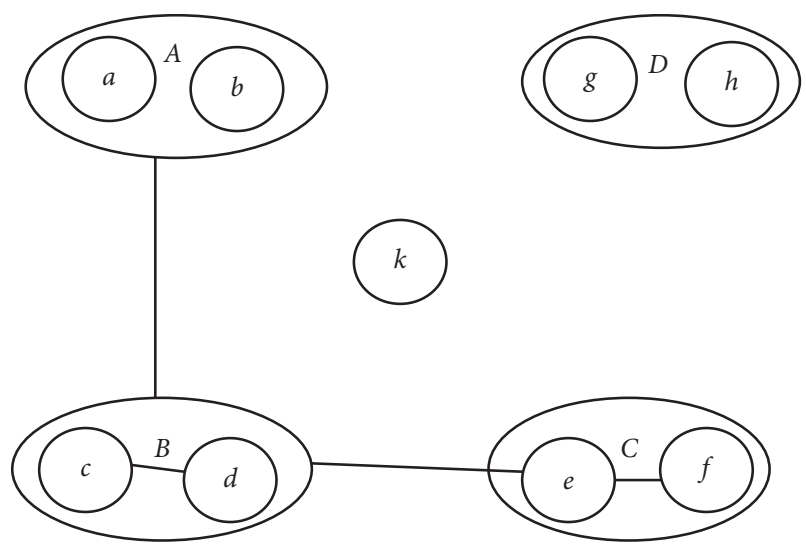

FIgURE 14: A cluster hypergraph with two isolated nodes.

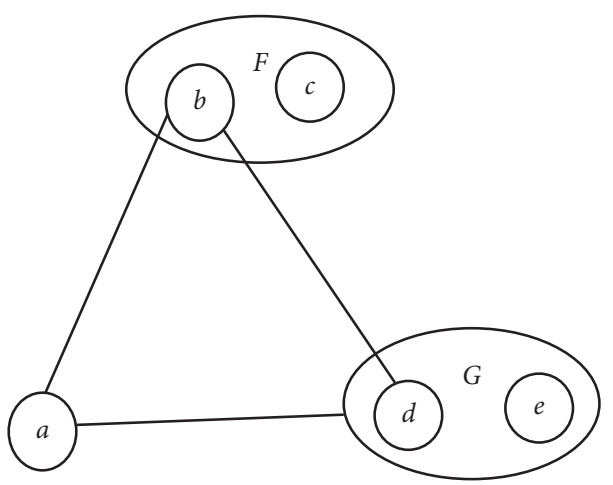

Figure 15: A cluster hypergraph with one isolated node. 


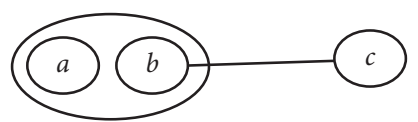

Figure 16: A cluster hypergraph $G$.

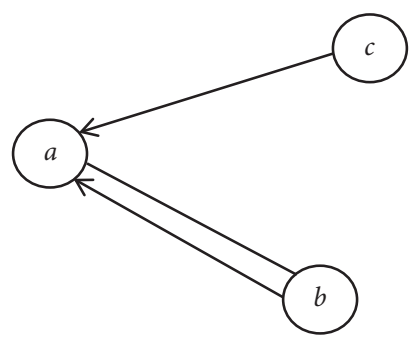

FIGURE 17: Corresponding semidirected graph of G.

Proposition 2. Competition number of a $(2,2)$-uniform cluster hypergraph is zero.

Proof. Consider a $(2,2)$ - uniform cluster hypergraph and draw the virtual presentation of it. Then, draw the bidirected lines between all nodes. Then, we observe that the semidirected graph is obtained whose corresponding competition cluster hypergraph is the $(2,2)$-uniform cluster hypergraph. Hence, there is no isolated node in the graph, and the competition number is zero. This completes the proof.

Theorem 4. Let $G$ be a semidirected graph and $G_{m}$ be the $m$ step semi directed graph of $G$, then $C\left(G_{m}\right)=C_{m}(G)$.

Proof. Since $\mathrm{G}$ is a semi-directed graph and $\mathrm{G}_{\mathrm{m}}$ is the $\mathrm{m}$-step semi-directed graph of $\mathrm{G}$. Then vertex set of both graphs is the same. Let $(u, v) \in C\left(G_{m}\right)$. Then, there exist edges $\left(\overrightarrow{u, x_{1}}\right)$, $\left(\overrightarrow{v, x_{1}}\right) ;\left(\overrightarrow{u, x_{2}}\right),\left(\overrightarrow{v, x_{2}}\right) ; \ldots,\left(\overrightarrow{u, x_{n}}\right) ;\left(\overrightarrow{v, x_{n}}\right)$ for some integer $n$. Now, $N^{+}(u) \cap N^{+}(v)=\left\{x_{1}, x_{2}, \ldots, x_{n}\right\}$. Since an edge $\left(\overrightarrow{u, x_{1}}\right) \in G_{m}$ implies there exists a path of length $m$ from $u$ to $x_{1}$ and similar for $\left(\overrightarrow{v, x_{1}}\right) \in G_{m}$; hence $(u, v) \in C_{m}(G)$. Similarly, if an edge $(x, y) \in C_{m}(G)$ then it implies $(x, y) \in C\left(G_{m}\right)$. Therefore, $C\left(G_{m}\right)=C_{m}(G)$.

Proposition 3. In a semidirected graph $G=\left(V, E_{1}, \overrightarrow{E_{2}}\right)$, if $m>|V|$, then $C_{m}(G)$ is a null cluster hypergraph.

Proof. Since $C_{m}(G)$ is an $m$-step competition cluster hypergraph of $G$ and if $m>|V|$, then there does not exist a path of length $m$ in $G$. Hence, $N_{m}^{+}(u)=N_{m}^{+}(v)=$ $\varnothing$ and $N_{m}^{+}(u) \cap N_{m}^{+}(v)=\varnothing$ for all $u, v \in G$. Thus, there does not exist any edge in $C_{m}(G)$. Therefore, $C_{m}(G)$ is a null cluster hypergraph.

\section{Application and Conclusions}

This study developed basic terminologies of mixed graphs. Some properties have been discussed. Another notion is that the cluster hypergraphs are also introduced. And finally, competition cluster hypergraphs of such semidirected graphs have been described. These types of competition
TABle 1: Top six COVID-19-affected countries (collected from Wikipedia dated 15 March 2020).

\begin{tabular}{lccc}
\hline Country & Total affected & Date of the first case & Affected from \\
\hline China & 81,218 & 31 December 2019 & \\
Italy & 69,176 & 31 January 2020 & China \\
USA & 54,935 & 19 January 2020 & China \\
Spain & 42,058 & 31 January 2020 & China \\
Germany & 33,593 & 25 February 2020 & Italy \\
Iran & 24,811 & 19 February 2020 & China \\
\hline
\end{tabular}

TABLE 2: Top 20 countries of carbon emissions (collected from Wikipedia dated 15 March 2020).

\begin{tabular}{lcc}
\hline Country & Total emissions & Per capita emissions \\
\hline China & $9.04 \mathrm{Bn}$ & 6.59 \\
United States & $5.00 \mathrm{Bn}$ & 15.53 \\
India & $2.07 \mathrm{Bn}$ & 1.58 \\
Russia & $1.47 \mathrm{Bn}$ & 10.19 \\
Japan & $1.14 \mathrm{Bn}$ & 8.99 \\
Germany & $729.77 \mathrm{Mn}$ & 8.93 \\
South Korea & $585.99 \mathrm{Mn}$ & 11.58 \\
Iran & $552.40 \mathrm{Mn}$ & 6.98 \\
Canada & $549.23 \mathrm{Mn}$ & 15.32 \\
Saudi Arabia & $531.46 \mathrm{Mn}$ & 16.85 \\
Brazil & $450.79 \mathrm{Mn}$ & 2.17 \\
Mexico & $442.31 \mathrm{Mn}$ & 3.66 \\
Indonesia & $441.91 \mathrm{Mn}$ & 1.72 \\
South Africa & $427.57 \mathrm{Mn}$ & 7.77 \\
United Kingdom & $389.75 \mathrm{Mn}$ & 5.99 \\
Australia & $380.93 \mathrm{Mn}$ & 15.83 \\
Italy & $330.75 \mathrm{Mn}$ & 5.45 \\
Turkey & $317.22 \mathrm{Mn}$ & 4.1 \\
France & $290.49 \mathrm{Mn}$ & 4.37 \\
Poland & $282.40 \mathrm{Mn}$ & 7.34 \\
\hline
\end{tabular}

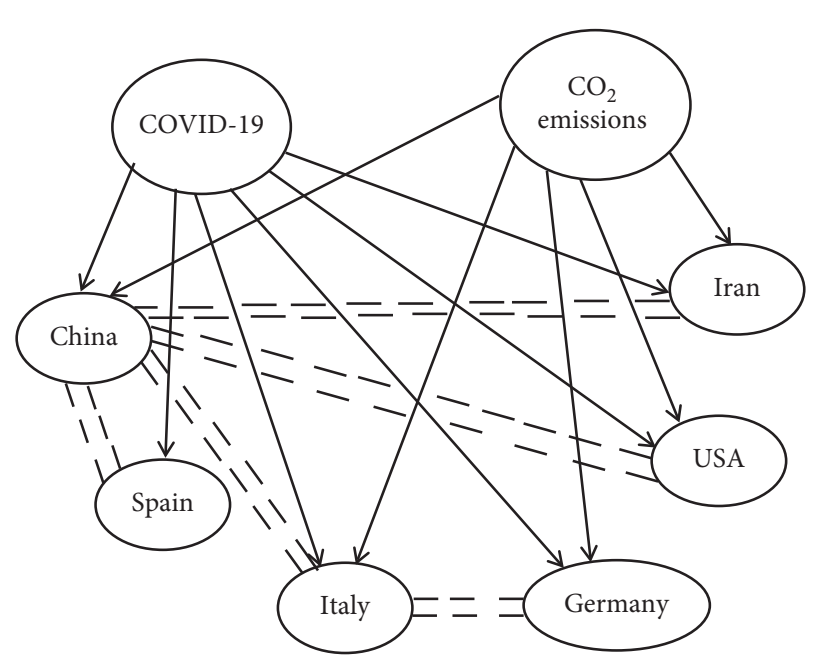

Figure 18: A semidirected graph.

graphs can be used to represent group competitions. A network is considered for COVID-19-affected areas as follows to show such a competition. 

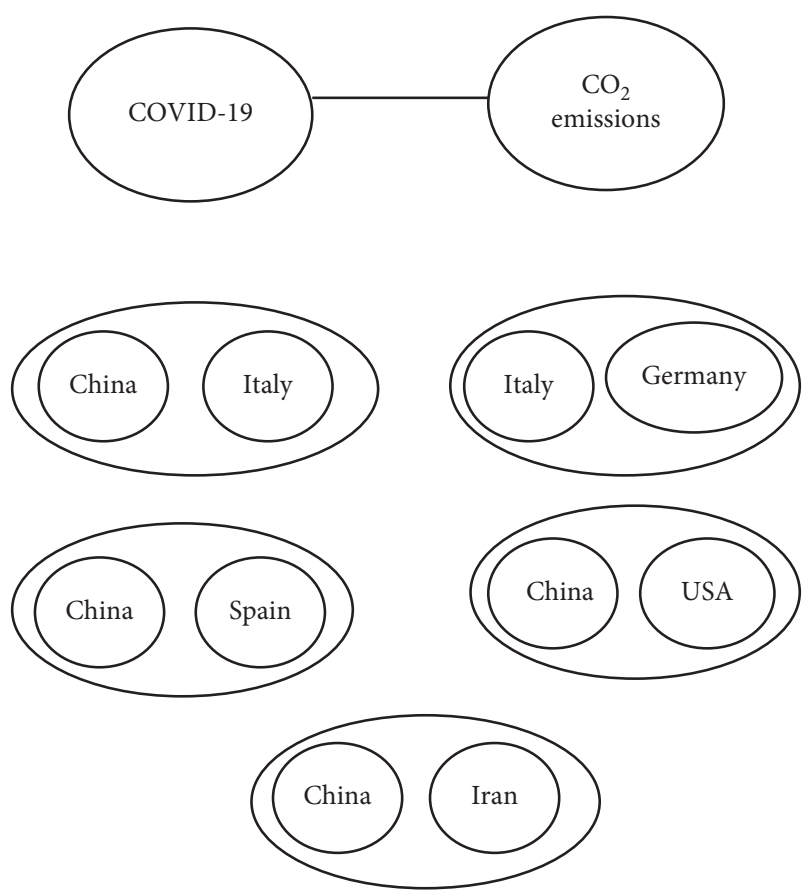

FIgURE 19: Competition cluster hypergraphs of Figure 18.

Affected places are assumed as nodes of a semidirected graph along with the source node as "COVID-19." Another different topic, major carbon emission countries are added in this network along with a source node as "carbon emission." Undirected edges connect the regions which are affected by COVID-19 through other countries. For Tables 1 and 2, the corresponding semidirected graph is shown in Figure 12, and its corresponding competition cluster hypergraph is shown in Figure 13.

The step-by-step process to find out the competition cluster hypergraphs is given as follows:

Step 1. Construction of Semidirected Graphs. Six highest COVID-19-affected countries have been assumed as nodes along with two fictitious nodes COVID-19 and $\mathrm{CO}_{2}$ emissions for a small semidirected graph. All the assumed countries are affected by COVID-19 and $\mathrm{CO}_{2}$ emissions. Thus, there will be direct edges from COVID-19 and $\mathrm{CO}_{2}$ emissions to all the nodes. If one country is affected by others, then there will be undirected edges (see Table 1 and Figure 18).

Step 2. Resultant Competition Hypergraphs. In the resultant competition hypergraphs, cliques will form cluster nodes. China-Italy, China-Spain, Italy-Germany, China-USA, and China-Iran are cluster nodes for this case (Figure 19). Between two nodes, there will be edges if the nodes have common out-neighbourhoods in semidirected graphs. Hence, the nodes COVID-19 and $\mathrm{CO}_{2}$ emissions will have one edge.

This small illustration can be extended to large networks for the detection of competition among different species, different countries, different cultures, etc., in several networks. Few related terms like $m$-step competition cluster hypergraphs and competition numbers have been analyzed with proper examples. This study will be a backbone for a new branch of hypergraphs and cluster hypergraphs along with competition cluster hypergraphs of semidirected graphs. Along with the theoretical developments, these theories may be applied to find out real-world competitions in business and sports industries where clusters are meaningful. The significant contributions of this study are given in Section 5.1.

\subsection{The Insights of the Research}

(i) A few properties of semidirected graphs have been established

(ii) Cluster hypergraphs are introduced

(iii) Competition cluster hypergraphs are defined

(iv) $m$-step competition cluster hypergraphs have been classified

(v) Various features of competition cluster hypergraphs have been studied

(vi) An application/numerical example of COVID-19 networks by using competition cluster hypergraphs has been given

\section{Data Availability}

The data, provided in the article, were collected from Wikipedia and https://www.worldometers.info/ coronavirus/\#countries. They are available in the public domain, so the authors have no restriction on that.

\section{Conflicts of Interest}

The authors declare that they have no conflicts of interest.

\section{References}

[1] J. E. Cohen, Interval Graphs and Food Webs: A Finding and a Problem, Document 17696-PR, RAND Corporation, Santa Monica, CA, USA, 1968.

[2] F. S. Roberts, "Food webs, competition graphs, and the boxicity of ecological phase space," in Theory and Applications of Graphs, Y. Alavi and D. Lick, Eds., pp. 477-490, SpringerVerlag, New York, NY, USA, 1978.

[3] F. S. Roberts, Discrete Mathematical Models, with Applications to Social, Biological, and Environmental Problems, PrenticeHall, Englewood Cliffs, NJ, USA, 1976.

[4] R. J. Opsut, "On the computation of the competition number of a graph," SIAM Journal on Algebraic Discrete Methods, vol. 3, no. 4, pp. 420-428, 1982.

[5] S. R. Kim, T. A. McKee, F. R. McMorris, and F. S. Roberts, "pcompetition graphs," Linear Algebra and Its Applications, vol. 217, pp. 167-178, 1995.

[6] S. R. Kim, T. A. McKee, R. R. McMorris, and F. S. Roberts, " $p$ competition number," Discrete Applied Mathematics, vol. 46, pp. 89-92, 1993.

[7] R. C. Brigham, F. R. McMorris, and R. P. Vitray, "Tolerance competition graphs," Linear Algebra and Its Applications, vol. 217, pp. 41-52, 1995. 
[8] H. H. Cho and S. R. Kim, "The competition number of a graph having exactly one hole," Discrete Mathematics, vol. 303, pp. 32-41, 2005.

[9] B. J. Li and G. J. Chang, "The competition number of a graph with exactly one hole, all of a which are independent," Discrete Applied Mathematics, vol. 157, pp. 1337-1341, 2009.

[10] K. A. S. Factor and S. K. Merz, "The (1,2)-step competition graph of a tournament," Discrete Applied Mathematics, vol. 159, pp. 100-103, 2011.

[11] C. Berge, Graphs and Hypergraphs, Elsevier, New York, NY, USA, 1973.

[12] C. Berge, Hypergraphs, North-Holland, Amsterdam, Netherlands, 1989.

[13] G. Burosch and P. V. Ceccherini, "A characterization of cubehypergraphs," Discrete Mathematics, vol. 152, no. 1-3, pp. $55-68,1996$.

[14] R. Bergen, S. Fallat, A. Gorr et al., "Infection in hypergraphs," Discrete Applied Mathematics, vol. 237, pp. 43-56, 2018.

[15] D. Z. Djokovic, "Distance preserving subgraphs of hypercubes," Journal of Combinatorial Theory, Series B, vol. 14, pp. 263-267, 1973.

[16] P. Frankl and G. Y. Katona, "Extremal $k$-edge-Hamiltonian hypergraphs," Electronic Notes in Discrete Mathematics, vol. 5, pp. 130-133, 2000.

[17] G. Y. Katona and H. A. Kierstead, "Hamiltonian chains in hypergraphs," Journal of Graph Theory, vol. 30, no. 3, pp. 205-212, 1999.

[18] B. Bollabos and A. D. Scott, "Judicious partitions of 3-uniform hypergraphs," European Journal of Combinatorics, vol. 21, pp. 289-300, 2000.

[19] R. I. Tyshkevich and V. E. Zverovich, "Line hypergraphs," Discrete Mathematics, vol. 161, no. 1-3, pp. 265-283, 1996.

[20] M. Sarwar, M. Akram, and N. O. Alshehri, "A new method to decision-making with fuzzy competition hypergraphs," Symmetry, vol. 10, no. 9, 2018.

[21] M. Akram, M. Sarwar, and R. A. Borzooei, "Certain network models using intuitionistic fuzzy hypergraphs," Journal of Intelligent \& Fuzzy Systems, vol. 35, no. 2, pp. 1905-1922, 2018.

[22] M. Sarwar and M. Akram, "Bipolar fuzzy circuits with applications," Journal of Intelligent \& Fuzzy Systems, vol. 34, no. 1, pp. 547-558, 2018.

[23] M. Sarwar and M. Akram, "Certain algorithms for computing strength of competition in bipolar fuzzy graphs," International Journal of Uncertainty, Fuzziness and Knowledge-Based Systems, vol. 25, no. 6, pp. 877-896, 2017.

[24] M. Akram and M. Sarwar, "Novel applications of $m$-polar fuzzy competition graphs in decision support system," Neural Computing and Applications, vol. 30, no. 10, pp. 3145-3165, 2018.

[25] M. Akram and A. Luqman, Fuzzy Hypergraphs and Related Extensions, Studies in Fuzziness and Soft Computing, Springer, Berlin, Germany, 2020.

[26] M. Hamidi and A. Borumand Saeid, "Achievable singlevalued neutrosophic graphs in Wireless sensor networks," New Mathematics and Natural Computation, vol. 14, no. 2, pp. 157-185, 2018.

[27] M. Hamidi and A. Borumand Saeid, "Creating and computing graphs from hypergraphs," Kragujevac Journal of Mathematics, vol. 43, no. 1, pp. 139-164, 2019.

[28] M. Akram, A. N. Gani, and A. B. Saeid, "Vague hypergraphs," Journal of Intelligent \& Fuzzy Systems, vol. 26, no. 2, pp. 647-653, 2014.
[29] M. Hamidi and A. Borumand Saeid, "On derivable trees," Transactions on Combinatorics, vol. 8, no. 2, pp. 21-43, 2019.

[30] M. Sonntag and H.-M. Teichert, "Sum numbers of hypertrees," Discrete Mathematics, vol. 214, no. 1-3, pp. 285-290, 2000.

[31] M. Sonntag and H.-M. Teichert, "Competition hypergraphs," Discrete Applied Mathematics, vol. 143, no. 1-3, pp. 324-329, 2004.

[32] N. V. Lambin and V. S. Tanaev, "On a circuit-free orientation of mixed graphs,” Dokladi Akademii Navuk BSSR, vol. 14, pp. 780-781, 1970 .

[33] Y. N. Sotskov and V. S. Tanaev, "Chromatic polynomial of a mixed graph," Vestsi Akademii Navuk BSSR, Seriya Fiz.-Mat Navuk, vol. 6, pp. 20-23, 1976.

[34] C. Adiga, B. R. Rakshith, and W. So, "On the mixed adjacency matrix of a mixed graph," Linear Algebra and Its Applications, vol. 495, pp. 223-241, 2016.

[35] J. Liu and X. Li, "Hermitian-adjacency matrices and Hermitian energies of mixed graphs," Linear Algebra Appl, vol. 466, pp. 182-207, 2015.

[36] A. M. Mohammed, "Mixed graph representation and mixed graph isomorphism," Journal of Science, vol. 30, no. 1, pp. 303-310, 2017

[37] C. C. Yang and Y. Lee, "Interactants and activities on facebook, instagram, and twitter: associations between social media use and social adjustment to college," Applied Developmental Science, vol. 24, no. 1, pp. 62-78, 2020. 